\title{
ERRATA 2
}

Na "Carta do Editor Convidado" (http://dx.doi.org/10.1590/S0104-59702020000100001) publicada no v.27, n.1 de História, Ciências, Saúde - Manguinhos, considerem-se as seguintes correções:

- Na página 7, nota 1 , onde se lia "Por isso, convidamos um editor adjunto da revista para apresentar um breve panorama da trajetória e relevância da história da saúde. Carlos Henrique Assunção Paiva faz uma reflexão, mais pertinente do que nunca, em um momento de desmoronamento da saúde pública brasileira.",

- leia-se "Por isso, convidamos o Observatório História e Saúde para apresentar um breve panorama da trajetória e relevância da história da saúde, mais pertinente do que nunca, em um momento de desmoronamento da saúde pública brasileira.".

- Na página 9, linha 5, onde se lia "brasileira.2",

- leia-se "brasileira.".

- Na página 9, linhas 19-23, onde se lia

"Carlos Henrique Assunção Paiva ${ }^{i}$

${ }^{i}$ Editor adjunto, pesquisador, Casa de Oswaldo Cruz/Fiocruz.

Rio de Janeiro - RJ - Brasil

orcid.org/0000-0002-7478-9628

carlos.paiva@fiocruz.br",

- leia-se

"Carlos Henrique Assunção Paiva ${ }^{i}$

${ }^{i}$ Pesquisador, Casa de Oswaldo Cruz/Fiocruz.

Rio de Janeiro - RJ - Brasil

orcid.org/0000-0002-7478-9628

carlos.paiva@fiocruz.br

Luiz Antônio Teixeira ${ }^{\mathrm{ii}}$

ii Pesquisador, Casa de Oswaldo Cruz/Fiocruz.

Rio de Janeiro - RJ - Brasil

orcid.org/0000-0001-8871-0928 
Fernando A. Pires-Alves ${ }^{\mathrm{iii}}$

iii Pesquisador, Casa de Oswaldo Cruz/Fiocruz.

Rio de Janeiro - RJ - Brasil

orcid.org/0000-0002-5091-9539

José Roberto Franco Reis ${ }^{\text {iv }}$

iv Pesquisador, Casa de Oswaldo Cruz/Fiocruz.

Rio de Janeiro - RJ - Brasil

orcid.org/0000-0002-1133-2778

Carlos Fidelis Ponte ${ }^{\mathrm{v}}$

v Pesquisador, Casa de Oswaldo Cruz/Fiocruz.

Rio de Janeiro - RJ - Brasil

orcid.org/0000-0003-1976-6287

Luiz Alves Araújo Neto ${ }^{\mathrm{vi}}$

${ }^{\text {vi }}$ Pesquisador, Casa de Oswaldo Cruz/Fiocruz.

Rio de Janeiro - RJ - Brasil

orcid.org/0000-0001-7965-2957".

- Na página 9, suprima-se a nota 2.

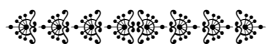

